\title{
Phase-Specific Motor Efference during a Rhythmic Motor Pattern
}

\author{
Ignacio Alonso, Agustín Sanchez Merlinsky, and Lidia Szczupak \\ Departamento de Fisiología, Biología Molecular y Celular, Facultad de Ciencias Exactas y Naturales, Universidad de Buenos Aires and Instituto de \\ Fisiología, Biología Molecular y Neurociencias, Ciudad Universitaria, (C1428EHA) Buenos Aires, Argentina
}

Neuronal circuits that control motor behaviors orchestrate multiple tasks, including the inhibition of self-generated sensory signals. In the hermaphroditic leech, $\mathrm{T}$ and $\mathrm{P}$ mechanosensory neurons respond to light touch and pressure on the skin, respectively. We show that the low threshold T cells were also sensitive to topological changes of the animal surface, caused by contraction of the muscles that erect the skin annuli. P cells were unresponsive to this movement. Annuli erection is part of the contraction phase of crawling, a leech locomotive behavior. In isolated ganglia, T cells showed phase-dependent IPSPs during dopamine-induced fictive crawling, whereas $\mathrm{P}$ cells were unaffected. The timing and magnitude of the T-IPSPs were highly correlated with the activity of the motoneurons excited during the contraction phase. Together, the results suggest that the central network responsible for crawling sends a reafferent signal onto the T cells, concomitant with the signal to the motoneurons. This reafference is specifically targeted at the sensory neurons that are affected by the movements; and it is behaviorally relevant as excitation of $\mathrm{T}$ cells affected the rhythmic motor pattern, probably acting upon the rhythmogenic circuit. Corollary discharge is a highly conserved function of motor systems throughout evolution, and we provide clear evidence of the specificity of its targets and timing and of the benefit of counteracting self-generated sensory input.

Key words: corollary discharge; leech; mechanosensory; rhythmic motor behaviour

\section{Significance Statement}

Neuronal circuits that control motor behaviors orchestrate multiple tasks, including inhibition of sensory signals originated by the animal movement, a phenomenon known as corollary discharge. Leeches crawl on solid surfaces through a sequence of elongation and contraction movements. During the contraction, the skin topology changes, affecting a subpopulation of mechanosensory receptors, $\mathrm{T}$ (touch) neurons, but not $\mathrm{P}$ (pressure) sensory neurons. In the isolated nervous system, T neurons were inhibited during the contraction but not during the elongation phase, whereas $\mathrm{P}$ cells were unaffected throughout crawling. Excitation of T cells during the contraction phase temporarily disrupted the rhythmic pattern. Thus, corollary discharge was target (T vs $\mathrm{P}$ ) and phase (contraction vs elongation) specific, and prevented self-generated signals to perturb motor behaviors.

\section{Introduction}

Neuronal circuits that control motor behaviors simultaneously orchestrate several tasks. In addition to determining the timely activation of multiple muscles, these circuits also prevent that self-generated sensory signals cause conflicting effects (Cullen, 2004; Crapse and Sommer, 2008). The somatic sensory system,

Received May 15, 2019; revised Jan. 14, 2020; accepted Jan. 15, 2020.

Author contributions: I.A., A.S.M., and L.S. performed research; I.A., A.S.M., and L.S. analyzed data; L.S. designed research; L.S. wrote the first draft of the paper; L.S. edited the paper; L.S. wrote the paper.

This work was supported by Agencia Nacional de Promoción Científica y Tecnológica Grant PICT 2016-2073 and Universidad de Buenos Aires Ciencia y Tecnología Grant 20020150100179 to L.S. We thank Marina Friszman for technical drawing; Licentiate Lirane Moutinho for technical assistance; and Drs. Violeta Medan, Graciela Kearney, Jutta Kretzberg, and Horacio Rotstein for highly valuable comments on the manuscript.

The authors declare no competing financial interests.

Correspondence should be addressed to Lidia Szczupak at szczupak@retina.ar.

https://doi.org/10.1523/JNEUROSCI.1201-19.2020

Copyright $\odot 2020$ the authors dedicated to process externally generated mechanical stimuli on the skin, may be a target of self-generated signals, as movements compress or stretch the skin (Tuthill and Wilson, 2016). It has been described that motor networks produce efferent copies of motor commands whose function is to cancel out self-generated sensory signals (von Holst and Mittelstaedt, 1950). The phenomenon is known as corollary discharge (Sperry, 1950; Crapse and Sommer, 2008).

Leeches count on two different sensors to process mechanical inputs onto the skin, that cover overlapping intensity ranges (Nicholls and Baylor, 1968; Kretzberg et al., 2016). In each segment, the corresponding ganglion includes three pairs of T cells sensitive to light touch and two pairs of $\mathrm{P}$ cells sensitive to pressure. The axon of each cell type reaches the periphery through ipsilateral roots and extend their terminals in the skin (Blackshaw, 1981). This population of mechanosensory neurons codes for intensity and location of mechanical stimuli (Lewis and 
i

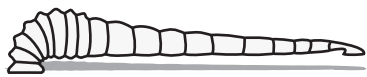

ii

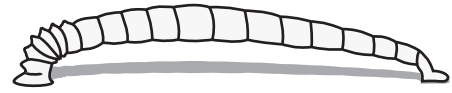

iii

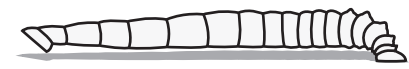

iv

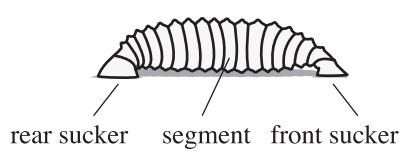

Figure 1. Drawing of four instances of a crawl step. Top to bottom, The leech initiates the elongation by (Phase i) detaching the front sucker from the ground while the rear sucker is still attached; (Phase ii) the leech attaches the front sucker after maximal elongation; (Phase iii) the leech starts the contraction by detaching the rear sucker; and (Phase iv) the leech ends the contraction when it reattaches the rear sucker. Suckers and midbody segments are identified in Phase iv. In Hirudo verbena, each segment includes 5 annuli; for simplicity, we included a single annulus to enhance the shift between flat (see Phases $\mathrm{i}$ and ii) annuli when the segment is extended to erected profiles (see Phase iv) when the segment contracts. Reprinted with permission from Stern-Tomlinson et al. (1986).

Kristan, 1998a,b; Pirschel and Kretzberg, 2016). While the role of these cells as exteroceptors has been widely documented, their role as proprioceptors has not been analyzed. In the present study, we show that the low threshold T cells are excited by topology changes of the skin, known as annuli erection, whereas $\mathrm{P}$ cells were unaffected by this skin stimulus.

Leech locomotion on solid ground is known as crawling. The crawling motor pattern consists basically of two phases: elongation of the body shifting the head to a distant forward point (Fig. 1, Phases $\mathrm{i}$ and ii), followed by contraction of the body moving the tail closer to the head (Fig. 1, Phases iii and iv), anchored on rear and front suckers, respectively. The elongation is executed by the activation of circular muscles, whereas the contraction depends on the activation of longitudinal muscles aided by erection of skin annuli (Gray et al., 1938; Eisenhart et al., 2000). Thus, there is room to expect that the network controlling crawling includes efferent signals directed toward $\mathrm{T}$ cells that counteract self-generated sensory activity during the contraction phase.

To analyze this question, we focused on the nervous system. Perfusion of leech isolated nerve cords, as well as single midbody ganglia, with dopamine induces rhythmic motor activity compatible with crawling (Puhl and Mesce, 2008; Bernardo PerezEtchegoyen et al., 2012). Typically, crawling is recognized by the rhythmic activity of motoneurons that excite longitudinal muscles (Fig. 1, contraction phase, Steps iii and iv) out of phase of excitors of circular muscles (Fig. 1, elongation phase, Steps i and ii) (Baader and Kristan, 1992; Baader, 1997). It is worth noting that the period and burst duration of crawling measured in intact animals (Stern-Tomlinson et al., 1986) and the dopamineinduced crawling in single isolated ganglia (Bernardo PerezEtchegoyen et al., 2012) exhibit comparable characteristics.

During dopamine-induced crawling, T cells receive inhibitory signals correlated with the contraction phase. $\mathrm{P}$ cells that were insensitive to skin topology changes remained unaffected. The phase-dependent inhibition of $\mathrm{T}$ cells appeared as behaviorally relevant since experimental excitation of these cells during the contraction phase shortened the duration of the phase and caused a phase advance.
This study performed in the leech reveals the specificity of the reafferent signal. The corollary discharge was not globally affecting the whole population of mechanosensors, but it was targeted at the neurons specifically affected by the animal movements, and at the specific phase at which the movement takes place. The results strongly suggest that the efferent signal was not generated by the motoneurons but was originated at a higher level, probably at the central pattern generator (CPG).

\section{Materials and Methods}

Biological preparation. Leeches (Hirudo sp) weighing 2-5 g were obtained from commercial suppliers (Leeches USA and Niagara Leeches) and maintained at $15^{\circ} \mathrm{C}$ in artificial pond water. These animals are hermaphrodites. The leech nervous system is composed of a chain of 21 midbody ganglia flanked by head and tail brains. Each midbody ganglion contains all the sensory and motor neurons that innervate the corresponding segment via root nerves (Muller et al., 1981).

Studies were performed in isolated body-wall preparations or in isolated single ganglia ranging from midbody ganglia $7-13$. The tissue was bathed in normal saline (in mM as follows: $115 \mathrm{NaCl}, 4 \mathrm{KCl}, 1.8 \mathrm{CaCl}_{2}, 1$ $\mathrm{MgSO}_{4}, 10 \mathrm{HEPES}, 10$ glucose, $\left.\mathrm{pH} 7.4\right)$ at room temperature $\left(20^{\circ} \mathrm{C}-\right.$ $25^{\circ} \mathrm{C}$ ) and pinned to Sylgard (Dow Corning) in a recording chamber. The sheath covering the ganglion was dissected away, leaving the neuronal cell bodies exposed to the external solution.

For body-wall preparations, the tubular body was cut along the dorsal midline, and three consecutive segments were isolated, linked to the ganglion that innervates the central segment; the ganglia innervating adjacent segments were removed. The body-wall was mounted with the skin surface upward; the pins held the preparation at regions of the body-wall that were not innervated by the central ganglion, and an incision was done at the ventral midline to uncover the ganglion (see Fig. 2, inset). To allow a certain degree of contraction, the body-wall was pinned down, avoiding excessive tension, but stretched enough to prevent movement artifacts during the recordings. That annuli erection took place when annulus erector $(\mathrm{AE})$ motoneuron was excited; the topology change was confirmed visually under the scope during the experiment.

In the isolated ganglion preparation, one or both dorsal posterior (DP) nerves were left attached for extracellular recordings.

Electrophysiological recordings and experimental design. Intracellular somatic recordings were made with microelectrodes pulled from borosilicate capillary tubing (FHC), filled with $3 \mathrm{~m}$ potassium acetate (resistance 20-40 $\mathrm{M} \Omega$ ). The electrodes were connected to an Axoclamp 2B amplifier (Molecular Devices) operating in bridge mode using well bridge-balanced electrodes. Extracellular activity was recorded from DP nerves using suction electrodes connected to a differential a.c. amplifier (Neuroprobe 1700, AM Systems). The intracellular and extracellular recordings were digitized using an analog digital converter (Digidata 1440, Molecular Devices) and acquired using a commercial program (Clampex 9.2, Molecular Devices) at a sampling rate of $5 \mathrm{kHz}$. The sensory T and P neurons and the AE motoneurons were readily recognized by their soma location and electrophysiological properties.

To evoke crawling, the ganglion was superfused with dopamine (Puhl and Mesce, 2008). The $75 \mu \mathrm{M}$ dopamine hydrochloride (Sigma Millipore) solution was prepared fresh at the beginning of each experimental day, and only one crawling episode was evoked per ganglion. The crawling motor pattern was monitored via extracellular recording of the DP nerve, where the largest spike corresponds to the excitor of dorsal longitudinal muscles, cell 3 (DE-3) (Ort et al., 1974). This cell is active during the contraction phase of crawling (Baader and Kristan, 1992; Baader, 1997).

Motoneurons in the leech extend the whole central arborizations within the ganglion where the soma is located; and the vast majority of these neurons, including $\mathrm{AE}$ and DE-3 motoneurons, extend the axon to the periphery through the contralateral lateral nerves. $\mathrm{T}$ and $\mathrm{P}$ sensory neurons present their major sensory field in the local ipsilateral hemisegment (where the soma is located), and they also extend neurites to anterior and posterior ganglia, from where they reach to the periphery and innervate secondary sensory fields. In the body-wall and the isolated 
ganglion preparations used in this series of experiments, the sensory neurons are confined to the local peripheral and central responses, respectively.

In two series of experiments, $T$ cells were stimulated during crawling episodes. To test the excitability of T cells (see Fig. $5 B, C$ ) during crawling, short pulses $(5 \mathrm{~ms}$ ) were applied at $1 \mathrm{~Hz}$, whose amplitude was enough to generate spikes at $\mathrm{T}$-cell baseline potential. To test the effect of bursts of T cells spikes during crawling (see Figs. 6-8), 2-3 s suprathreshold pulses were injected while the crawling behavior was monitored in the computer display; pulses were applied in two conditions: in between DE-3 bursts or once a DE-3 burst was initiated. At least three cycles before and after the excitatory pulse were left unaffected to obtain prepulse and a postpulse crawling fragments.

Data analysis and statistics. Data analysis was performed using commercial software (Clampfit 9, Molecular Devices and Axograph 1.5.4). Spikes in DP nerves and in AE and T cells were detected using amplitude threshold. To measure AE firing frequency, a low pass filtered trace was subtracted from the original trace. To summarize T-cell response to annuli erection in body-wall preparations, we measured the number of $\mathrm{T}$ spikes during the excitation of AE neurons for each cell and plotted these values as a function of AE firing frequency. From these plots, we calculated the response corresponding to an AE firing frequency of $10 \mathrm{~Hz}$ and expressed the responses of each cell relative to this value.

Crawling was characterized by the cycle period, measured as the time elapsed between the first DE-3 spikes in two successive bursts; duration of bursts was measured as the time between the first and last spike in a burst; and the firing frequency, calculated as the number of spikes in a burst divided by the burst duration. More specific analysis is detailed in the corresponding figure legends.

Unless otherwise stated, data are presented as mean \pm SEM. Curve fitting (least square method) and statistical analysis were performed using commercial software (Kaleidagraph 3.0.2, Abelbeck Software). Onesample Wilcoxon signed rank test was used to compare data with a fixed value $(p<0.05)$. In each case, we detail the number of ganglia used and the number of animals from which those ganglia were obtained.

\section{Results}

\section{Mechanosensory neurons act as proprioceptors}

Mechanosensory $\mathrm{T}$ and $\mathrm{P}$ cells are sensitive to skin indentation caused by external objects; $\mathrm{T}$ cells have been defined as low threshold because a light touch on the skin excites them, whereas P cells are excited by higher pressure (Nicholls and Baylor, 1968). To evaluate whether these sensory cells are sensitive to selfgenerated changes in skin topology, we isolated a fragment of the body-wall together with the ganglion that controls its sensorymotor functions (see Materials and Methods; Fig. 2A). To manipulate the skin topology, one of the bilateral pair of $\mathrm{AE}$ motoneurons was excited, as activation of these cells consistently causes erection of the skin annuli of the contralateral hemisegment (Rodriguez et al., 2004). T and P cells, contralateral to the stimulated AE motoneurons, were intracellularly recorded to monitor their activity.

Figure $2 A$ reveals that, in a body-wall preparation, injection of excitatory current pulses in an AE motoneuron caused excitation of the recorded $\mathrm{T}$ cell. Consistent with the fact that $\mathrm{T}$ cells are fast-adapting neurons (Nicholls and Baylor, 1968; Carlton and McVean, 1995), the responses to sustained AE firing, and thus to sustained annuli erection (Rodriguez et al., 2004), adapted rapidly, showing a drastic decrease in the firing frequency with time. Increasing the amplitude of the current pulses led AE to fire at increasing frequencies, and the number of $\mathrm{T}$-cell spikes increased as a linear function of the motoneuron firing frequency (Fig. $2 B$, top). To compare with previously published data that relate annuli indentation (as height of the annulus) with AE firing frequency (Rodriguez et al., 2004), we processed the old data in the same way as the current data (expressing the annuli height rela- tive to those corresponding to an $\mathrm{AE}$ firing frequency of $10 \mathrm{~Hz}$; Fig. $2 B$, bottom). Together, Figure $2 B$ shows that annuli erection and $\mathrm{T}$-cell firing increase linearly within a similar AE firing range.

When the same stimulation protocol was performed in isolated ganglia (in the absence of the body-wall), T cells did not fire a single action potential (data not shown; $n=22$ AE-T pairs, 7 ganglia, 4 animals), although $\mathrm{AE}$ fired at the same frequency range as in the presence of body-wall $(8-40 \mathrm{~Hz})$; and at the largest current pulse, the mean firing frequency was of $25 \pm 2 \mathrm{~Hz}$. This result indicates that the excitation of T cells was not due to central synaptic connections between the motoneuron and the sensory neuron, but required the presence of the periphery, strongly suggesting that it was generated by skin indentation.

Experimental protocols as described in Figure $2 \mathrm{~A}$ were performed to evaluate the effect of annuli erection on the mechanosensory $\mathrm{P}$ cells. Figure $2 \mathrm{C}$ shows that the membrane potential of $\mathrm{P}$ cells did not exhibit any change during annuli erection.

Together, the results indicate that the low threshold mechanosensory T cells, which are sensitive to external tactile stimuli, were also sensitive to self-generated mechanical signals, and therefore they acted as proprioceptors. On the other hand, P cells, sensitive to pressure on the skin, were not affected by annuli erection.

Mechanosensory cell activity in the context of motor patterns Although T cells are weak activators of motor responses (Kristan, 1982), they bear electrical synaptic connections with motoneurons (Nicholls and Purves, 1970, 1972); thus, their activation by self-generated mechanical signals during crawling could affect the progression of the behavior. In consequence, it was of interest to evaluate whether the neuronal network responsible of crawling involves T cells.

Motor control results from the interaction of commands imparted by the nervous system onto the periphery, and feedback from the latter. However, it has been clearly documented in several species, including leeches, that the core of the rhythmic generator is located in the CNS; and the rhythmogenic circuits can be analyzed in the absence of the periphery and even in specific compartments of the nervous system (Orlovsky et al., 1999). In such preparations, the motor output is recorded through intracellular or extracellular recordings of the motoneurons, which are useful proxies of the behavior (usually identified as "fictive behavior"). The validity of this approach has been previously demonstrated in rhythmic motor behaviors in the leech, including swimming (Kristan et al., 1974; Kristan and Calabrese, 1976; Stent et al., 1978), crawling (Stern-Tomlinson et al., 1986; Baader, 1997; Eisenhart et al., 2000), and blood circulation (Thompson and Stent, 1976; Calabrese, 1977; Calabrese and Peterson, 1983), which have been monitored in progressively reduced settings: intact animals, semi-intact preparations, and the isolated nervous system. In all these cases, there is a striking homology between motor output in the absence of the periphery and the correlation with behavior. This includes dopamineinduced crawling, in which the rhythmic motor output is compatible with the characteristics of crawling as analyzed in semi-intact preparations (Puhl and Mesce, 2008; Bernardo Perez-Etchegoyen et al., 2012). Study of circuits in the isolated nervous system allows an in-depth analysis of nervous circuits that would be highly limited in more complete configurations.

We studied the T-cell involvement in crawling in the isolated midbody ganglia to evaluate the existence of interactions between the rhythmogenic circuit and this sensory neuron. If T-cell firing contributes to the progression of crawling, the neuronal network is not expected to interfere with T-cell activity, allowing it to 
A
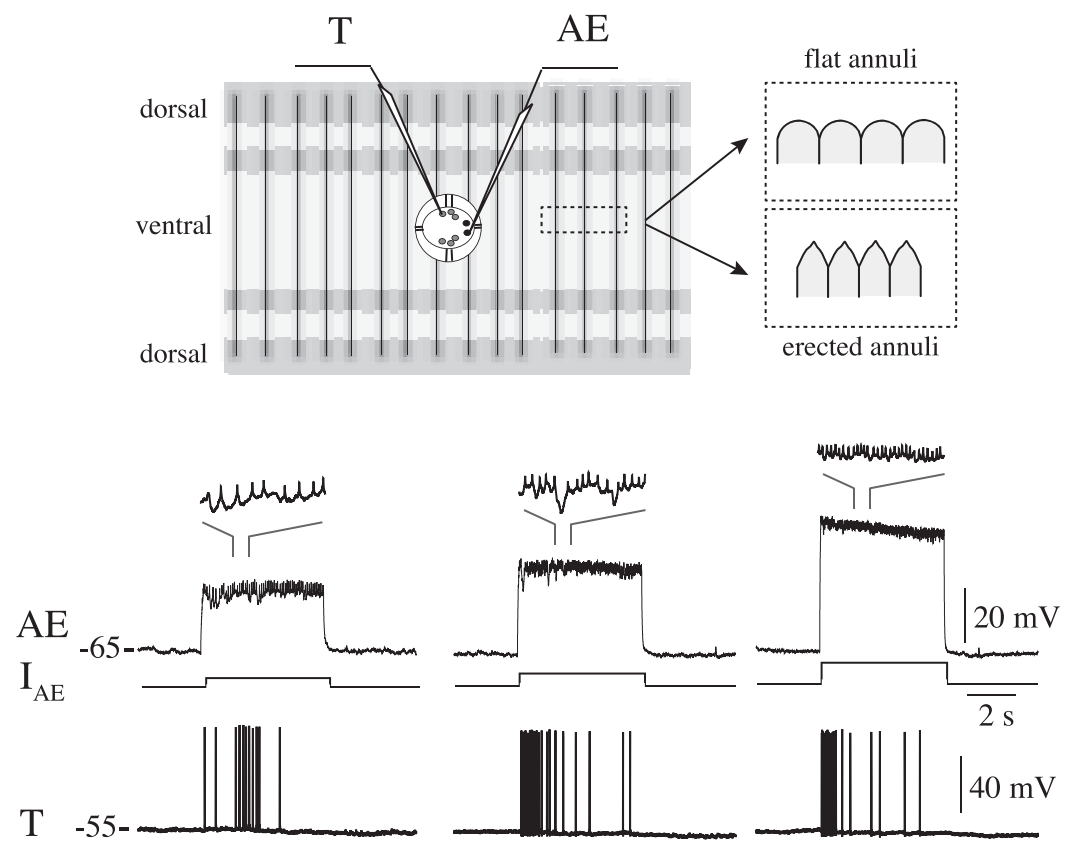

B
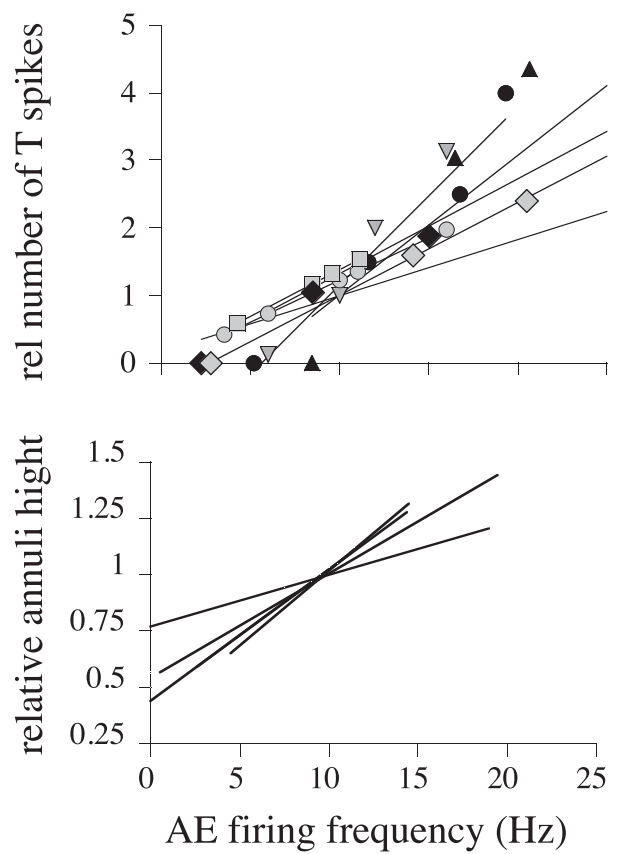

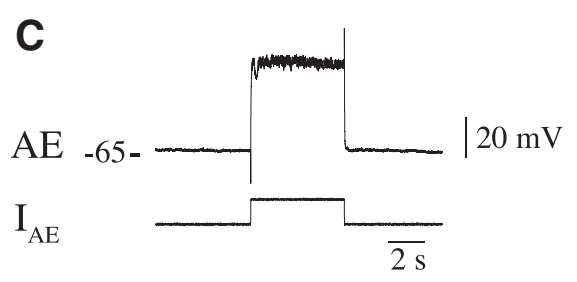

P -50

$15 \mathrm{mV}$

Figure 2. T cell as a proprioceptive sensor. $A$, The top drawling represents the experimental scheme: a piece of body-wall comprising three segments was isolated with the ganglion innervating the central segment attached (see Materials and Methods). Dorsal and ventral body regions are specified. A hole was open on the ventral region to expose the ventral side of the ganglion where AE and $\mathrm{T}$ cells were recorded intracellularly. Arrows point to sketches of a piece of the skin displaying two profiles: flat and erected annuli, caused by silencing and exciting an AE motoneuron, respectively. Bottom, Recordings of AE and T cells while square current pulses $\left(I_{A E}\right)$ of increasing amplitude (0.5-1.25 nA) were injected in the AE cell. In the leech, somatic recordings of motoneuron spikes result from passive propagation of events generated at electrically distant sites. On top of the AE recordings, a temporal expansion reveals individual spikes. $\boldsymbol{B}$, Top, The number of spikes recorded in T cells as a function of AE firing frequency. The number of spikes during the AE pulse is expressed relative to the values corresponding to an AE firing of $10 \mathrm{~Hz}$ (see Materials and Methods) in each preparation. Each symbol represents individual T-AE pairs ( $n=7$ T-AE pairs, 3 preparations in 3 animals). Lines indicate linear fit (for the 7 experiments $p<0.03$ and $R>0.89$ ). Bottom, The height of the annuli as a function of AE firing frequency as published by Rodriguez et al. (2004), but made relative to the $10 \mathrm{~Hz}$ AE firing frequency to match the analysis of T responses. Only linear fits are shown (the four fits had a $p<0.02$ and $R>0.78)$. C, Recordings of AE and $P$ cells while a square current pulse $(1.25 \mathrm{nA})$ was injected in the AE cell. The experimental setting is as described by $A$, but recording $P$ instead of $T$ cells. Identical results were obtained in 6 pairs of $P$ and $A E$ cells, tested in 4 body-wall preparations from 3 animals. $A, C$, The numbers on the left of the recordings indicate the membrane potential in $\mathrm{mV}$.

respond to proprioceptive signals; but if these signals impair the progression of the motor program, it is expected that the neuronal network prevents $\mathrm{T}$-cell excitation. If the first alternative applies, $\mathrm{T}$ membrane potential should be unaffected during crawling, that would enable self-generated excitation in the intact animal; while if the second alternative is right, we would expect $\mathrm{T}$ cell to be inhibited during crawling, and in particular during the contraction phase, at which annuli are erected (Gray et al., 1938; Eisenhart et al., 2000) that would counteract self-generated excitation in the intact animal.

Figure $3 A$ shows a fragment of a crawling episode, obtained in an isolated ganglion, where both DP nerves and a T cell were simultaneously recorded. The large spikes in each DP form solid bursts that showed a tight bilateral correlation, consistent with the fact that crawling is a symmetrical behavior. These DP bursts mark the contraction phase of crawling. T cells exhibited barrages of IPSPs that were mainly in phase with the DE-3 bursts. To evaluate the degree of correlation between the DE-3 bursts and the barrage of T-IPSPs, we identified the middle spike in each DE- 3 burst and the middle IPSP in the barrage(s) found within each cycle (Fig. 3B). The midpoint in each signal was chosen because it appears as the most reliable temporal variable; the beginning of the DE-3 burst or the T-IPSP can exhibit a larger variability. As shown by the polar histogram, the T-IPSPs were temporally correlated with the DE-3 bursts ( 0 degrees in the polar histogram), and anticipated the motoneuron activity by $\sim 5 \%$ of the cycle period. Considering the 253 cycles analyzed (13 ganglia, 6 leeches), $82 \%$ were temporally correlated with a T-IPSPs, and $26 \%$ of the cycles showed more than one barrage of IPSPs per cycle.

$P$ cells, which were not affected by annuli erection, showed no membrane potential oscillations during crawling (Fig. $3 C$ ), indicating that $\mathrm{T}$ cells were specific targets of the reafferent signal. 


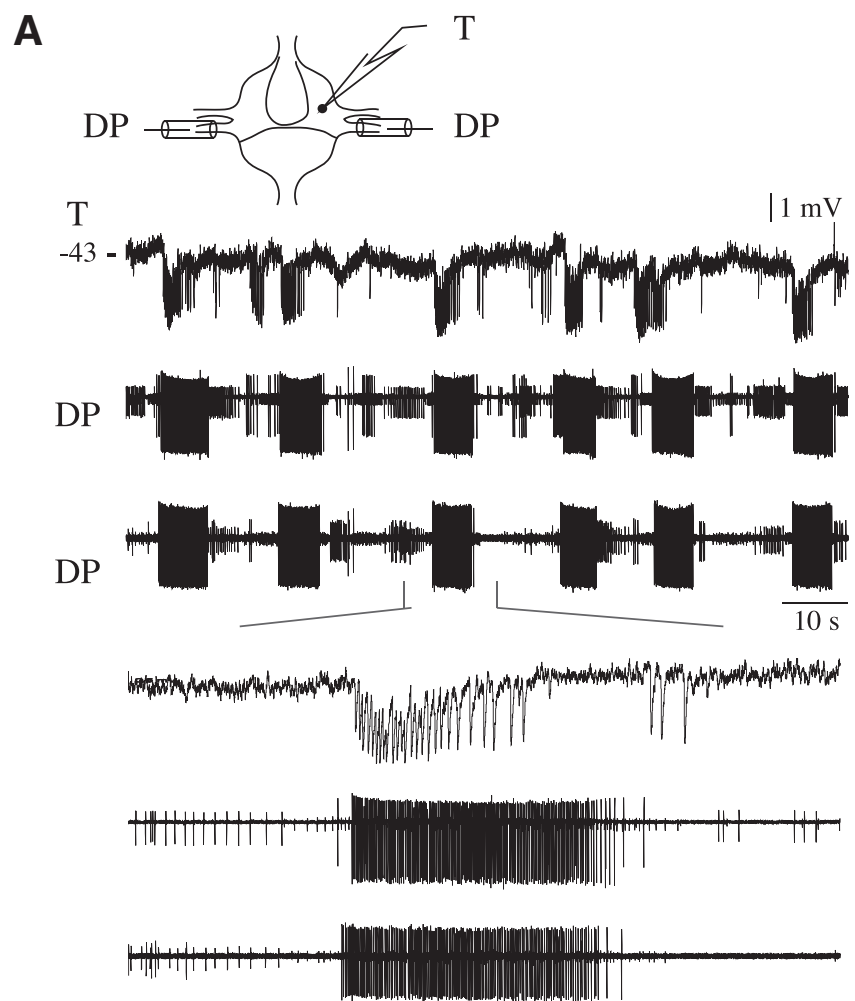

B
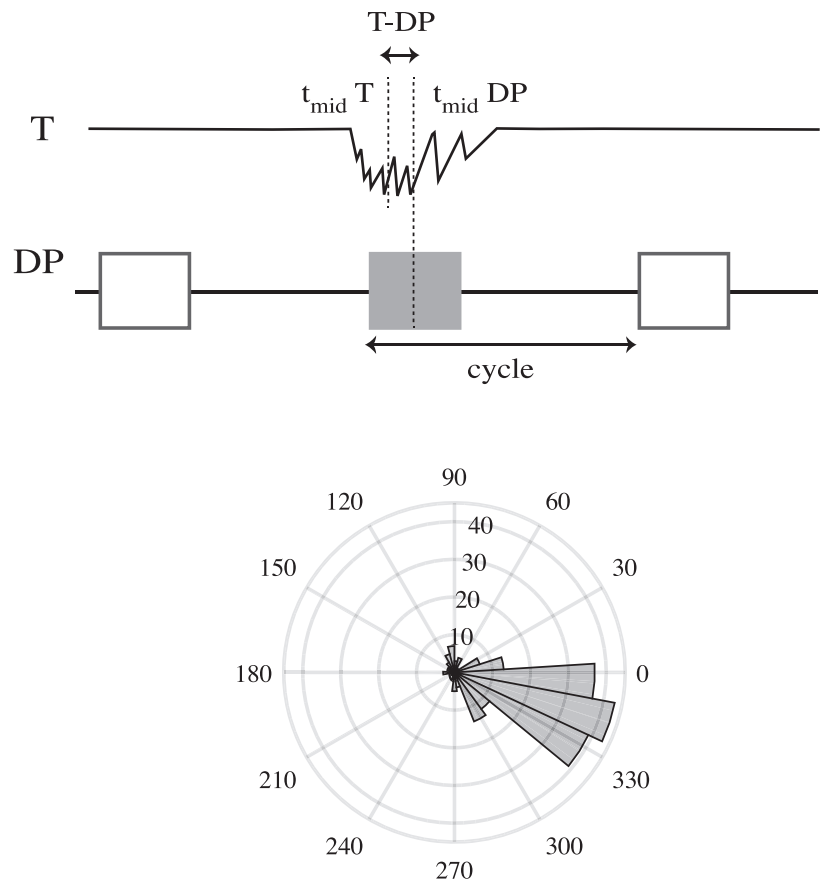

C

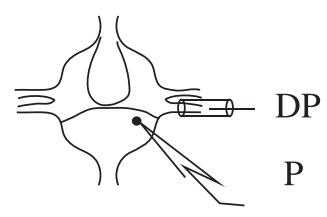

$1 \mathrm{mV}$

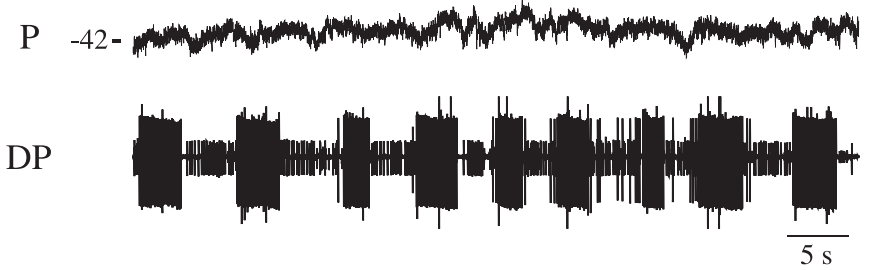

Figure 3. T cells were inhibited during the contraction phase of crawling. A, Inset, experimental setting: in an isolated ganglion, T cells were intracellularly recorded while motoneuron activity was recorded extracellularly in the pair of DP nerves. The recordings show the activity in T and DP in the course of a crawling episode. The largest spike in the DP nerve corresponds to the DE-3 motoneuron. A temporal expansion of the segment indicated by dashed lines is presented below. $\boldsymbol{B}$, Inset, Correlation analysis. White and gray boxes represent DE- 3 bursts; for simplicity, the T-IPSP corresponding to the gray box is the only one drawn. Right vertical dashed line indicates the middle spike of the gray DE-3 burst ( $\left.t_{\text {mid }} D P\right)$. Left line indicates the peak of the middle T-IPSP ( $\left.t_{\text {mid }} T\right)$. The difference between these two values $\left(\mathrm{t}_{\text {mid }} \mathrm{DP}-\mathrm{t}_{\text {mid }} \mathrm{T}\right)$ divided by the cycle period was used to estimate the correlation described in the polar histogram ( $n=253$ cycles in $13 \mathrm{ganglia}$, 6 leeches). The mean period in these experiments was $15 \mathrm{~s}(14.5 \pm 0.6 \mathrm{~s})$. C, Inset, experimental setting: in an isolated ganglion, $P$ cells are intracellularly recorded while motoneuron activity is recorded extracellularly in one DP nerve. The recordings show the activity in $\mathrm{P}$ and DP in the course of a crawling episode. Identical results were obtained in 8 pairs of $P$ and DP recordings, tested in 4 ganglia from 4 leeches; these studies included dorsal and ventral $P$ cells. $A, C$, The numbers on the left of the intracellular recordings indicate the membrane potential in $\mathrm{mV}$.

To evaluate whether T-IPSPs were conveyed by electrical or chemical synapses, the T-cell membrane potential was shifted to different values in the course of a crawling episode. As expected from a chemical synapse, the amplitude and polarity of T-IPSPs varied with the membrane potential, and their reversal potential was of $\sim-60 \mathrm{mV}$ (Fig. 4).

As it can be observed in Figure 3A, IPSPs were mostly generated in conjunction with DE-3 bursts, but some synaptic events took place in between DE-3 bursts. To evaluate the correspondence between the corollary discharge and the motoneuron activity level Figure $5 A$ describes the relationship between the magnitude of the synaptic responses of $\mathrm{T}$ cells and the firing frequency in DE-3 bursts. Null frequency $(0 \mathrm{~Hz})$ corresponds to
T-IPSPs that took place in between DE-3 bursts, rather than in conjunction with a burst. The graph reveals that synaptic events not associated with DE-3 bursts were of low magnitude (isolated IPSPs or the summation of very few IPSPs), and there is a tight correlation between the firing frequency of DE-3 and the magnitude of the simultaneously occurring T-IPSPs.

In somatic recordings, the IPSPs are reflected as deviations in the membrane potential of small amplitude. To evaluate whether these IPSPs affected the excitability of T cells, we applied short 0.5 $\mathrm{nA}$ pulses at $1 \mathrm{~Hz}$ along crawling episodes through the recording electrode. As shown in the example presented in Figure $5 B$, the pulses elicited spikes along the baseline, but when they occurred during the IPSP the depolarization failed to generate a spike. To 


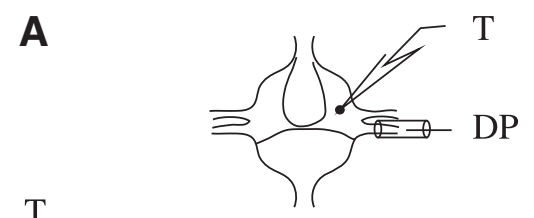

$\mathrm{T}$
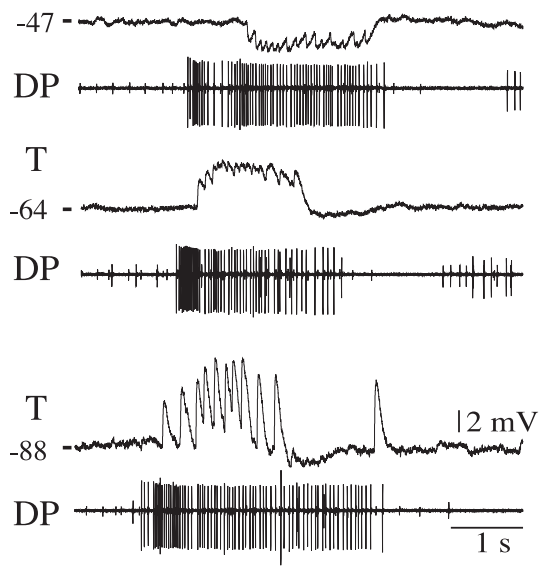

B
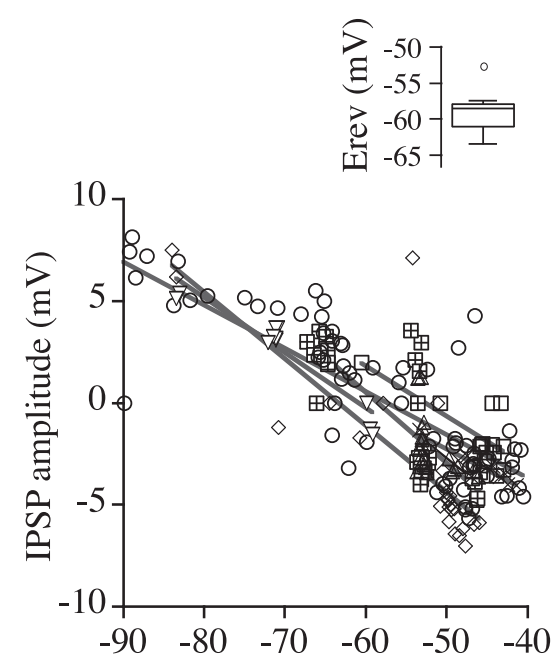

$\mathrm{T}$ membrane potential $(\mathrm{mV})$

Figure 4. The IPSP reversal potential. $\boldsymbol{A}$, Three sets of simultaneous recordings in a T cell and a DP nerve during a crawling episode, as the membrane potential of the T cell was shifted to different values indicated on the left in $\mathrm{mV}$. $\boldsymbol{B}$, The graph represents the IPSP amplitude as a function of the membrane potential of the T cell ( $n=6$ ganglia, 3 leeches). The T-IPSPs were measured as the area under the curve divided by the integration time. Lines indicate the linear fit $(p<0.02)$ of individual crawling episodes. Inset, Box plot of the reversal potential calculated from the linear fits ( $x$ at $y=0)$.

quantify the effect of the IPSPs on the excitability of $\mathrm{T}$ cells, considering that the pulses could fall at the edges or in the middle of the IPSPs, as well as along the baseline, we related each pulse to the potential relative to baseline. At the expense of underestimating such potential deviations, a low pass filter of the $T$-cell $\left(T_{f}\right)$ recording was used, and in which the average membrane potential of the recording was used as the reference $(0 \mathrm{mV})$ to measure the potential deviations $\left(\Delta V \mathrm{~m}_{\mathrm{f}}\right)$. It is worth noting that $\Delta \mathrm{Vm}_{\mathrm{f}}$ is not only an underestimation of the somatic recording but of the synaptic input at its site of origin in the neurites of $\mathrm{T}$ cells. Figure $5 \mathrm{C}$ shows the probability that a $\mathrm{T}$-pulse elicited a spike as a function of $\Delta \mathrm{Vm}_{\mathrm{f}}$, indicating that the negative shifts from baseline markedly decreased the excitability of T cells.

\section{Excitation of mechanosensory $\mathrm{T}$ cells impairs leech crawling in a phase-specific mode}

The results presented so far indicate that $\mathrm{T}$ cells are subjected to reafferent signals that prevent these mechanosensory neurons to fire during the contraction phase; this is consistent with the fact that $\mathrm{T}$ cells perform as proprioceptors, sensitive to selfgenerated signals evoked by movements inherent to this motor phase. The question that arises from this observation is what would be the consequence if $\mathrm{T}$ cells fired during crawling. Because the reafferent signal was phase-specific, we focused the analysis on two temporal windows: (1) in between bursts and (2) during a DE- 3 burst. To this aim, we applied excitatory pulses (2-3 nA, 1-3 s) onto T cells (T-pulses) in the course of crawling episodes in these two temporal windows.

Application of T-pulses in between DE-3 bursts caused no significant effect on the cycle period, the duration of the DE-3 bursts, or on the firing frequency of the motoneuron (Fig. 6). Application of such pulses as DE-3 was firing cut short the ongoing DE-3 burst in both DP nerves (Fig. 7A). The duration of the ongoing DE- 3 burst was approximately half that of the preceding bursts (Fig. $7 B, C$ ). The effect of T-pulses during the DE-3 burst was phasic as the rhythmic pattern following the intervention preserved the same properties as previous to it (Fig. 7D).

These results indicate that $\mathrm{T}$-pulses affected the ongoing activity of DE-3 neurons. The question that arises from this observation is whether T-pulses acted directly on the motoneuron or via the rhythmogenic circuit. To answer this question, we analyzed the cycle period during the intervention and compared it with the period before the intervention (Fig. 8). T-pulses in between DE-3 bursts had no effect on the ongoing cycle, showing the same period as the cycles previous to the pulse (Fig. 8A); but T-pulses applied during a DE-3 burst shortened the ongoing cycle: the DE-3 burst following the interrupted one was phase advanced (Fig. 8B).

Together, these results indicate that excitation of $\mathrm{T}$ cells during the contraction phase exhibited a phasic effect on crawling that decreased the ongoing DE-3 burst and cycle period.

\section{Discussion}

\section{Phase-specific corollary discharge onto $\mathrm{T}$ cells}

$\mathrm{T}$ cells are mechanosensory neurons, sensitive to deformation of the skin by light touch and water movement over the skin (Nicholls and Baylor, 1968; Rodriguez et al., 2004; Kretzberg et al., 2016), and in particular to velocity of indentation (Carlton and McVean, 1995). Here we show that these exteroceptors performed also as proprioceptors since they were activated by annuli erection induced by motor activity. It has been already suggested that the division between exteroceptive and proprioceptive functions needs to be revised (Tuthill and Wilson, 2016; Chagnaud et al., 2017), as neurons can display such dual function, as reported here in the leech and observed in Aplysia (Borovikov et al., 2000).

Given the fact that $\mathrm{T}$ cells were sensitive to annuli erection, and that this movement is part of the contraction phase of crawling (Eisenhart et al., 2000), the self-generated excitation could deliver misleading information during this motor phase, if interpreted as an external object touching the skin. We have found that the rhythmogenic network responsible of crawling delivered inhibitory inputs onto $\mathrm{T}$ cells that could counteract proprioceptive signals produced during the contraction phase of crawling. T-IPSPs were phase-specific, taking place during the contraction phase. The IPSPs exhibited a reversal potential of $-60 \mathrm{mV}$, suggesting an increase in chloride conductance (Lent, 1977) that decreased T-cell excitability (Fig. 5B,C). However, one should take into consideration that the actual inhibitory power of the IPSPs cannot be fully deduced from our studies. On one hand, the somatic recordings might underestimate the amplitude of the IPSPs at their site of origin, and the spikes elicited from the periphery may be affected differently than those generated centrally in our experiments. The effect of the corollary discharge on the input/output function of $\mathrm{T}$ cells highly depends on the site at which the inhibitory synapse impinges their neuritic arbor, lim- 
A

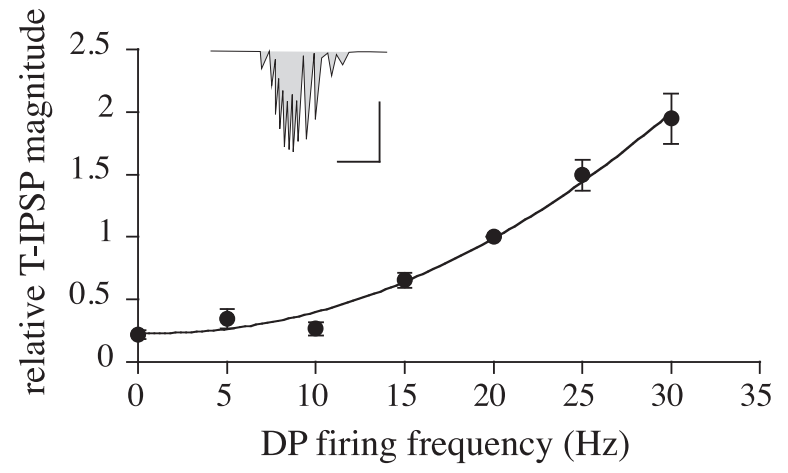

B
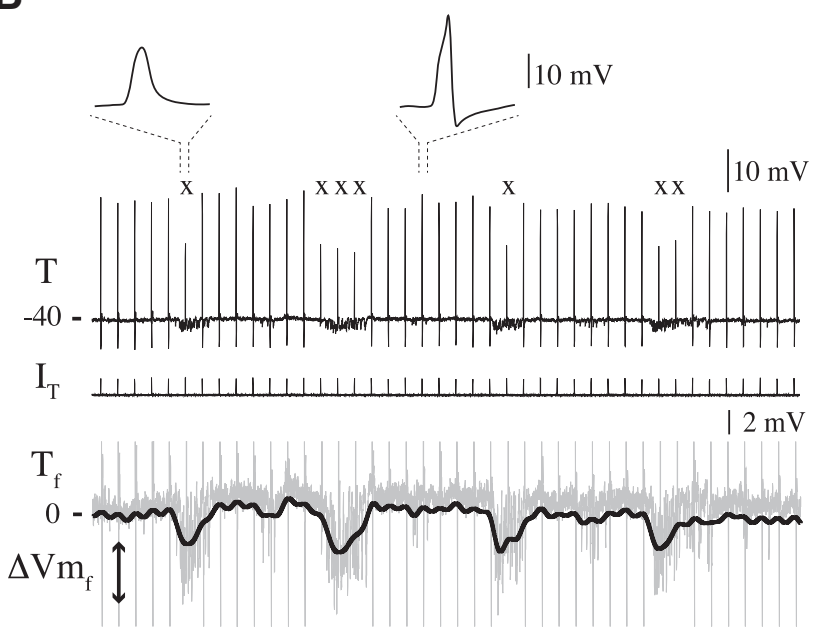

DP

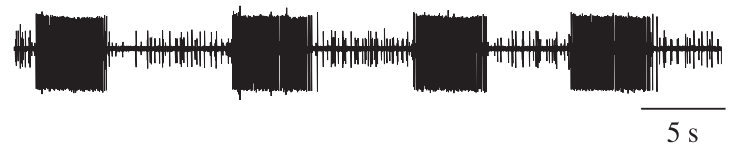

C

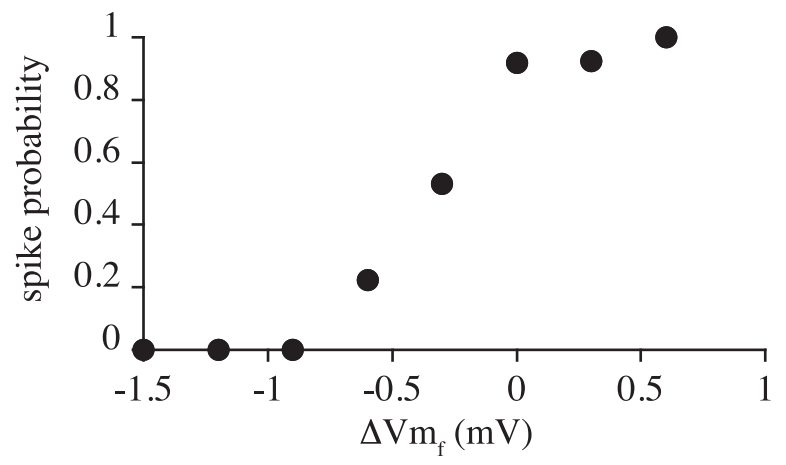

Figure 5. Magnitude and power of the T-IPSPS. A, Graph represents the magnitude of the IPSPs as a function of the firing frequency of the corresponding DP burst. The T-IPSPs ( $n=243$ cycles from 13 ganglia from 9 leeches) were measured as the area under the curve as shown in the inset (vertical and horizontal scales: $2 \mathrm{mV}$ and $1 \mathrm{~s}$, respectively); and are expressed relative to the mean IPSP magnitude correlated with a DE-3 firing frequency of $20 \mathrm{~Hz}$ in each crawling episode. $0 \mathrm{~Hz}$ corresponds to IPSPs generated in between DE-3 bursts. $\boldsymbol{B}$, Top, Bottom, Simultaneous activity of a T cell and a DP nerve, respectively, in the course of a crawling episode, as 5 ms pulses $\left(\mathrm{I}_{\mathrm{T}}, 0.5 \mathrm{nA}\right)$ were injected in the T cell at $1 \mathrm{~Hz}$. The amplitude of the pulses was adjusted to generate a spike when T was at its baseline (in between IPSPS). The number on the left of the intracellular recordings indicates the membrane potential in $\mathrm{mV}$; $x$ indicates the pulses that did not result in a T spike. Inset above traces, Temporal expansion of the events enclosed in the dashed lines, showing a subthreshold depolarization (left) and a spike (right), clearly distinguishable by their respective amplitudes and the lack of the typical T afterhyperpolarization. Underneath the T-cell recording, we show, in gray, an amplitude amplification of this trace; and superimposed, the thick line indicates the low $(0.5 \mathrm{~Hz})$ pass filtered trace $\left(\mathrm{T}_{\mathrm{f}}\right)$ of the recording.
A

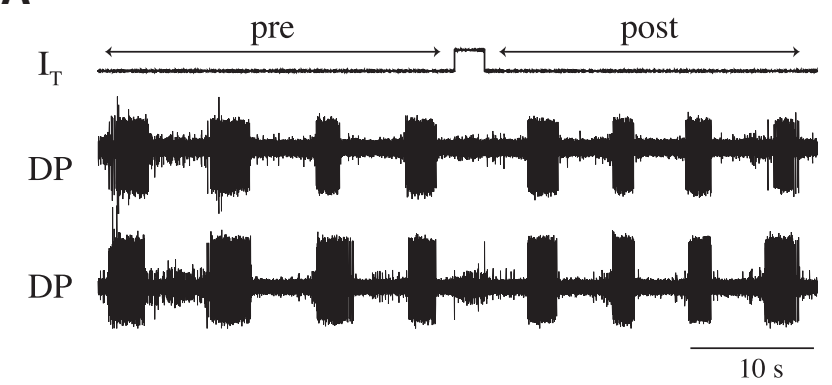

B
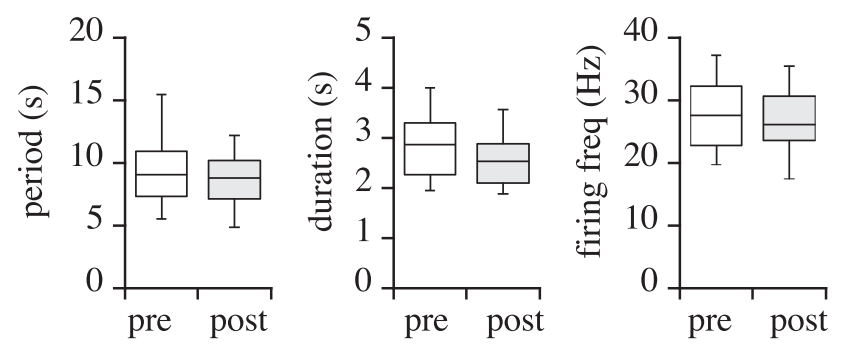

Figure 6. Excitation of T cells in between DE-3 bursts. $\boldsymbol{A}$, Extracellular recording of both DP nerves of a ganglion in the course of a crawling episode, as a barrage of spikes was elicited in the T cell (data not shown) by the injection of a $2 \mathrm{nA}$ pulse $\left(\mathrm{I}_{\mathrm{T}}\right)$, applied in between two consecutive DE-3 bursts. $B$, Box plots represent the magnitude of the cycle period, DE-3 burst duration, and DE-3 firing frequency in the "pre" and "post" fragments indicated in $\boldsymbol{A}$ ( $n=11$ pulses in 6 crawling episodes in 3 leeches).

iting the spike propagation from the periphery through the central branches of the sensory cell.

In addition to the phase specificity, the efferent signal was target specific. The mechanosensory P cells, which were insensitive to annuli indentation, remained unaffected by the widespread neuronal activity implicated during crawling, supporting the view that the corollary discharge onto $\mathrm{T}$ cells was not part of a generalized inhibition onto mechanosensory neurons innervating the skin, but was adjusted in a behaviorally relevant manner, to undermine possible self-generated mechanosensory signals.

\section{Origin of the corollary discharge}

The reafferent signal can originate at different levels along the motor circuit hierarchy (Poulet and Hedwig, 2007). The data do not support a direct effect of motoneurons on T cells. Excitation of AE motoneurons did not produce any response in $\mathrm{T}$ cells when tested in the isolated ganglia, indicating that the motoneurons did not drive the T-IPSPs. T and DE-3 soma are located on opposite sides of the ganglion; therefore, a thorough synaptic interaction could not be tested directly, but the recordings show that T-IPSPs slightly preceded DE-3 bursts, strongly suggesting that DE-3 firing was not the signal that caused the T-IPSPs. These observations are not sufficient to rule out the possibility that motoneuron activity is the origin of the corollary discharge but strongly undermine it. According to our interpretation, the marked correlation between the magnitude of the reafferent signal in $\mathrm{T}$ cells and the signal that drives the contraction phase (Fig. $5 A$ ) suggests that both signals originate in a common source. We propose that this source is the CPG present in each segment

The average membrane potential of the trace was subtracted from it. $\boldsymbol{C}$, Graph represents the probability that a T-pulse generated a spike as a function of the change in $\mathrm{T}_{\mathrm{f}}(n=264$ pulses, in 4 ganglia from 3 leeches). 
A

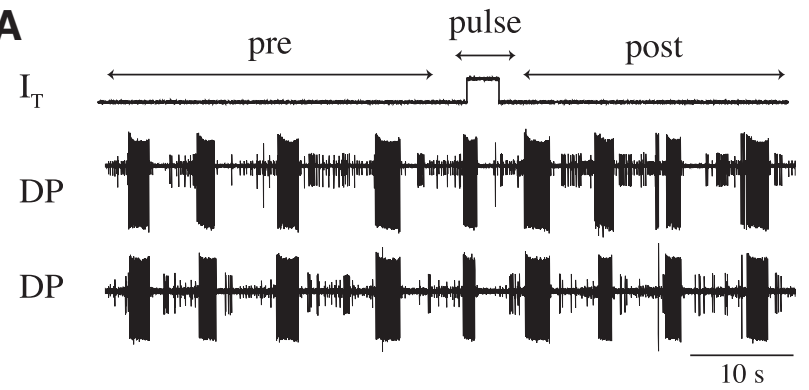

B

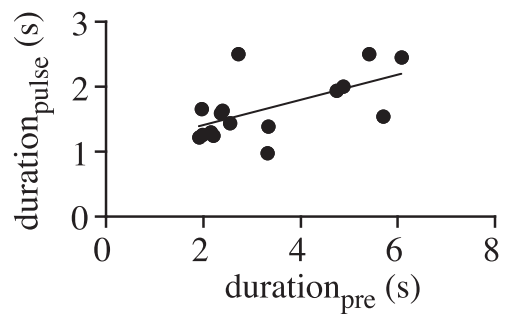

D
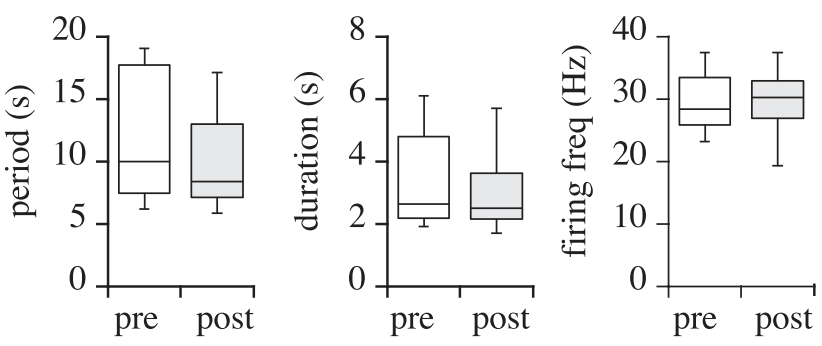

Figure 7. Excitation of T cells during a DE-3 burst. $A$, Extracellular recording of both DP nerves of a ganglion in the course of a crawling episode, as a barrage of spikes was elicited in the T cell (data not shown) by the injection of a $2 \mathrm{nA}$ pulse $\left(\mathrm{I}_{\mathrm{T}}\right)$, applied during a DE-3 burst. $\boldsymbol{B}$, Graph represents the relationship between the duration of the $D E-3$ burst during the pulse and duration of the bursts preceding it (mean of three preceding cycles). Line indicates a linear fit ( $r=$ $0.60, p=0.015)$. C, Box plot represents the relative magnitude of the duration of the DE- 3 burst during the pulse (relative to the corresponding mean of the "pre" and "post" values to each pulse). ${ }^{*} p<0.0001$, significantly different from 1.D, Box plots represent the magnitude of the cycle period, DE- 3 burst duration, and DE-3 firing frequency in the "pre" and "post" fragments indicated in $\boldsymbol{A} . \boldsymbol{B}-\boldsymbol{D}, n=16$ pulses in 6 crawling episodes in 4 leeches.

(Cacciatore et al., 2000; Puhl and Mesce, 2008; Rodriguez et al., 2012), reaching each target through parallel pathways.

\section{Functional role of the corollary discharge}

The existence of a corollary discharge signal suggests that T-cell excitation in the course of crawling may affect the normal progression of the motor pattern. Indeed, excitation of T cells during the contraction phase shortened the duration of the ongoing DE-3 burst and the corresponding cycle period.

Based on previous data, finding that excitation of $\mathrm{T}$ cells inhibited DE-3 firing (Fig. 7A-C) was unexpected. T cells are electrically coupled to excitors of longitudinal muscles ( $\mathrm{L}$ motoneurons), which are electrically coupled to DE-3 motoneurons (Ort et al., 1974; Bernardo Perez-Etchegoyen et al., 2012). Thus, the expected effect of T-cell excitation is to excite DE-3 neurons. Instead, we found that T-cell excitation cut short the DE-3 burst. This result and the fact that T-pulses affected the cycle period of crawling suggest that T-cell excitation acted upon the rhythmogenic circuit. As summarized in Figure 9, we propose two possible mechanisms that explain the effect of T-cell excitation: activation of the mechanosensory neurons curtails the input onto DE-3 (1) by exciting the elongation unit(s) or (2) by inhibiting the con-
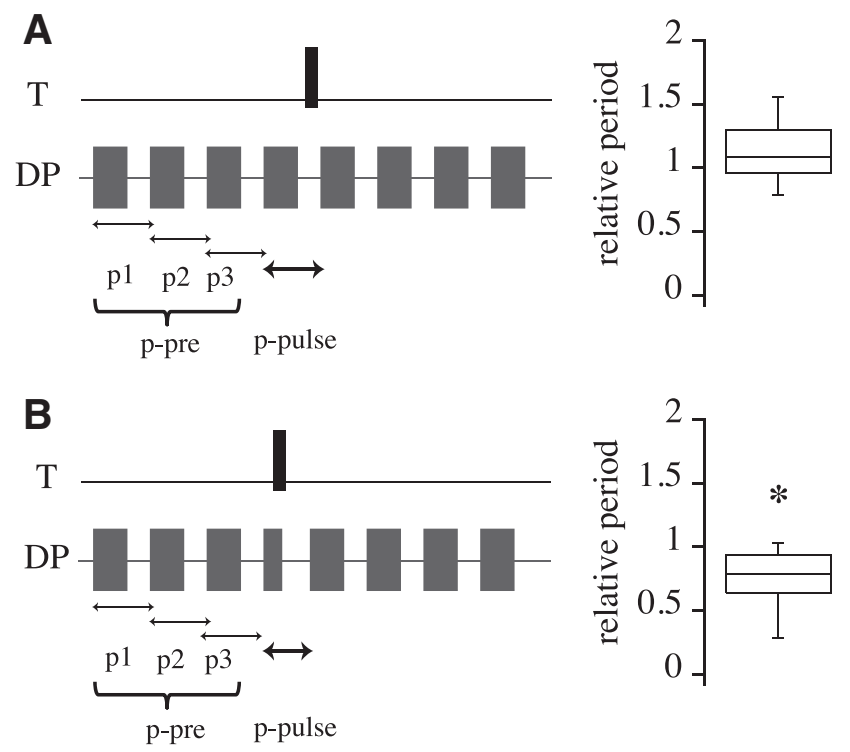

Figure 8. Excitation of T cells during a DE-3 burst caused a phase advance. $\boldsymbol{A}$, The scheme shows how we analyzed the effect of the T-pulse on the rhythmic activity of DE-3, when applied in between two DE-3 bursts. Two variables were measured: the mean of the three cycle periods previous to the pulse ( $p$-pre) and the period between the burst before the pulse and the burs after the pulse (p-pulse). The relative period is $p$-pulse/p-pre. Box plot represents the magnitude of the relative period for this protocol ( $n=11$ pulses in 6 crawling episodes in 4 animals). The values are not significantly different from 1 ( $p=0.23)$. $\boldsymbol{B}$, Same as in $\boldsymbol{A}$, but in this case the p-pulse is measure between the burst affected by the pulse and the following pulse ( $n=16$ pulses in 6 crawling episodes in 4 leeches). ${ }^{*} p<0.0001$, significantly different from 1.

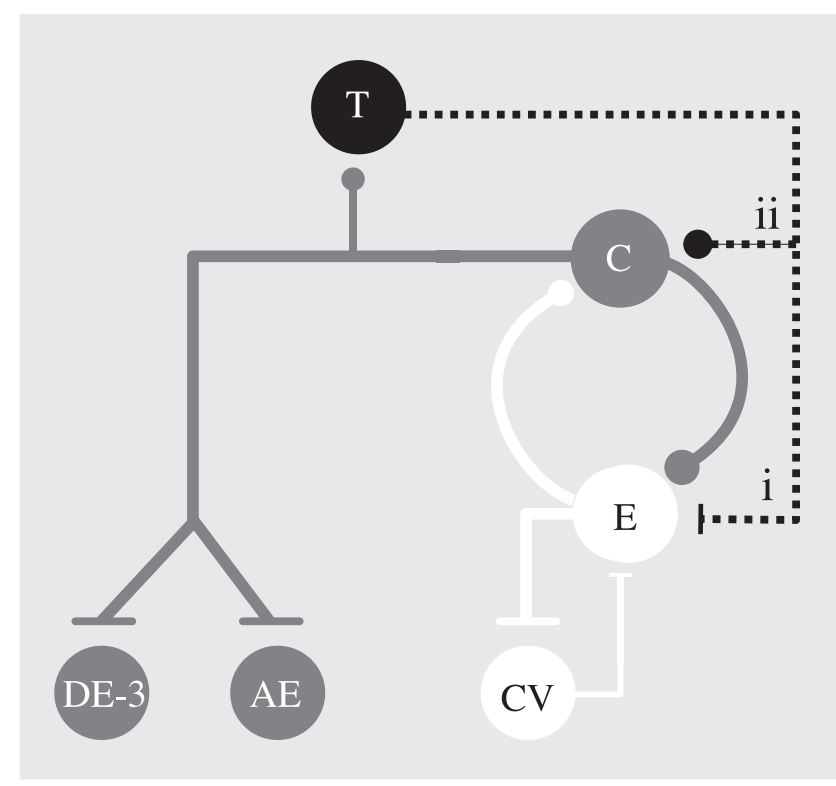

Figure 9. Putative configuration of the motor network. The drawing presents a putative connectivity diagram. Each segmental ganglion contains a CPG that controls (at least) two phases: contraction $(C)$ and elongation (E). C excites the motoneurons that control longitudinal muscles (e.g., DE-3) and AE motoneurons, whereas E excites circular muscles (e.g., excitors of circular ventral muscles [CV]), and it also extends an inhibitory connection to the mechanosensory $T$ cells. T cells are connected to the CPG in two alternative ways (dotted lines): (i) it excites $\mathrm{E}$; or (ii) it inhibits $\mathrm{C}$.

traction unit(s) of the CPG that controls crawling. Excitation of the elongation unit(s) would inhibit the contraction unit(s) and stop the excitatory input onto DE-3, whereas inhibition of the contraction unit(s) would release the elongation unit(s) from 
inhibition and cause an early initiation of an elongation phase. The lack of effect of T-cell excitation during the elongation phase is consistent with both interpretations, as during this phase the elongation unit(s) are already excited and the contraction unit(s) already inhibited. A direct evaluation of this connectivity pattern requires more information on the properties of the $\mathrm{CPG}$, which is presently unavailable.

Recently, we have shown that excitation of motoneurons active during the elongation phase ( $\mathrm{CV}$ motoneuron) interrupts the DE-3 burst and causes a phase advance (Rotstein et al., 2017). As indicated in Figure 9, we interpret that CV feeds back onto the CPG, exciting the unit(s) that controls its own activation, and thus inhibits the activity of the unit(s) that controls the activity of DE-3. This positive feedback from the motoneuron to the CPG was shown to influence the phase and period of crawling (Rotstein et al., 2017). Whether such feedback exists between DE-3 and the CPG remains to be studied.

Corollary discharge as a general control mechanism is a highly conserved function of motor systems throughout evolution (Poulet and Hedwig, 2007; Crapse and Sommer, 2008). Phasedependent inhibition of mechanosensory neurons during rhythmic motor programs has been observed in invertebrates and vertebrates (Kirk, 1985; Gossard and Rossignol, 1990; Wolf and Burrows, 1995; Seki et al., 2003; Chagnaud et al., 2015). The present description in the leech nervous system highlights that corollary discharge acts specifically upon behaviorally relevant sensory targets and evaluates the functional consequences of allowing the self-generated sensory input to prevail.

\section{References}

Baader AP (1997) Interneuronal and motor patterns during crawling behavior of semi-intact leeches. J Exp Biol 200:1369-1381.

Baader AP, Kristan WB (1992) Monitoring neuronal activity during discrete behaviors: a crawling, swimming and shortening device for tethered leeches. J Neurosci Methods 43:215-223.

Blackshaw SE (1981) Morphology and distribution of touch cell terminals in the skin of the leech. J Physiol 320:219-228.

Borovikov D, Evans CG, Jing J, Rosen SC, Cropper EC (2000) A proprioceptive role for an exteroceptive mechanoafferent neuron in Aplysia. J Neurosci 20:1990-2002.

Cacciatore TW, Rozenshteyn R, Kristan WB Jr (2000) Kinematics and modeling of leech crawling: evidence for an oscillatory behavior produced by propagating waves of excitation. J Neurosci 20:1643-1655.

Calabrese RL (1977) The neural control of alternate heartbeat coordination states in the leech, Hirudo medicinalis. J Comp Physiol A Neuroethol Sens Neural Behav Physiol 122:111-143.

Calabrese RL, Peterson E (1983) Neural control of heartbeat in the leech, Hirudo medicinalis. Symp Soc Exp Biol 37:195-221.

Carlton T, McVean A (1995) The role of touch, pressure and nociceptive mechanoreceptors of the leech in unrestrained behavior. J Comp Physiol A Neuroethol Sens Neural Behav Physiol 177:781-791.

Chagnaud BP, Banchi R, Simmers J, Straka H (2015) Spinal corollary discharge modulates motion sensing during vertebrate locomotion. Nat Commun 6:7982.

Chagnaud BP, Engelmann J, Fritzsch B, Glover JC, Straka H (2017) Sensing external and self-motion with hair cells: a comparison of the lateral line and vestibular systems from a developmental and evolutionary perspective. Brain Behav Evol 90:98-116.

Crapse TB, Sommer MA (2008) Corollary discharge across the animal kingdom. Nat Rev Neurosci 9:587-600.

Cullen KE (2004) Sensory signals during active versus passive movement. Curr Opin Neurobiol 14:698-706.

Eisenhart FJ, Cacciatore TW, Kristan WB (2000) A central pattern generator underlies crawling in the medicinal leech. J Comp Physiol A 186:631-643.

Gossard JP, Rossignol S (1990) Phase-dependent modulation of dorsal root potentials evoked by peripheral nerve stimulation during fictive locomotion in the cat. Brain Res 537:1-13.
Gray J, Lissmann HW, Pumphrey RJ (1938) The mechanism of locomotion in the leech (Hirudo medicinalis Ray). J Exp Biol 15:408-430.

Kirk MD (1985) Presynaptic inhibition in the crayfish CNS: pathways and synaptic mechanisms. J Neurophysiol 54:1305-1325.

Kretzberg J, Pirschel F, Fathiazar E, Hilgen G (2016) Encoding of tactile stimuli by mechanoreceptors and interneurons of the medicinal leech. Front Physiol 7:506.

Kristan WB (1982) Sensory and motor neurones responsible for the local bending response in leeches. J Exp Biol 96:161-180.

Kristan WB Jr, Calabrese RL (1976) Rhythmic swimming activity in neurones of the isolated nerve cord of the leech. J Exp Biol 65:643-668.

Kristan WB, Stent GS, Ort CA (1974) Neuronal control of swimming in the medicinal leech: I. Dynamics of the swimming rhythm. J Comp Physiol 94:97-119.

Lent CM (1977) The Retzius cells within the central nervous system of leeches. Prog Neurobiol 8:81-117.

Lewis JE, Kristan WB Jr (1998a) Representation of touch location by a population of leech sensory neurons. J Neurophysiol 80:2584-2592.

Lewis JE, Kristan WB Jr (1998b) A neuronal network for computing population vectors in the leech. Nature 391:76-79.

Muller KJ, Nicholls JG, Stent GS (1981) Neurobiology of the leech. Cold Spring Harbor, NY: Cold Spring Harbor Laboratory.

Nicholls JG, Baylor DA (1968) Specific modalities and receptive fields of sensory neurons in CNS of the leech. J Neurophysiol 31:740-756.

Nicholls JG, Purves D (1970) Monosynaptic chemical and electrical connexions between sensory and motor cells in the central nervous system of the leech. J Physiol 209:647-667.

Nicholls JG, Purves D (1972) A comparison of chemical and electrical synaptic transmission between single sensory cells and a motoneurone in the central nervous system of the leech. J Physiol 225:637-656.

Orlovsky GN, Deliagina TG, Grillner S (1999) Neuronal control of locomotion: Oxford: Oxford UP.

Ort CA, Kristan WB, Stent GS (1974) Neuronal control of swimming in the medicinal leech. J Comp Physiol A Neuroethol Sens Neural Behav Physiol 94:121-154.

Bernardo Perez-Etchegoyen C, Alvarez RJ, Rodriguez MJ, Szczupak L (2012) The activity of leech motoneurons during motor patterns is regulated by intrinsic properties and synaptic inputs. J Comp Physiol A Neuroethol Sens Neural Behav Physiol 198:239-251.

Pirschel F, Kretzberg J (2016) Multiplexed population coding of stimulus properties by leech mechanosensory cells. J Neurosci 36:3636-3647.

Poulet JF, Hedwig B (2007) New insights into corollary discharges mediated by identified neural pathways. Trends Neurosci 30:14-21.

Puhl JG, Mesce KA (2008) Dopamine activates the motor pattern for crawling in the medicinal leech. J Neurosci 28:4192-4200.

Rodriguez MJ, Iscla IR, Szczupak L (2004) Modulation of mechanosensory responses by motoneurons that regulate skin surface topology in the leech. J Neurophysiol 91:2366-2375.

Rodriguez MJ, Alvarez RJ, Szczupak L (2012) Effect of a nonspiking neuron on motor patterns of the leech. J Neurophysiol 107:1917-1924.

Rotstein HG, Schneider E, Szczupak L (2017) Feedback signal from motoneurons influences a rhythmic pattern generator. J Neurosci 37:9149-9159.

Seki K, Perlmutter SI, Fetz EE (2003) Sensory input to primate spinal cord is presynaptically inhibited during voluntary movement. Nat Neurosci 6:1309-1316.

Sperry RW (1950) Neural basis of the spontaneous optokinetic response produced by visual inversion. J Comp Physiol Psychol 43:482-489.

Stent GS, Kristan WB Jr, Friesen WO, Ort CA, Poon M, Calabrese RL (1978) Neuronal generation of the leech swimming movement: an oscillatory network of neurons driving a locomotory rhythm has been identified. Science 200:1348-1357.

Stern-Tomlinson W, Nusbaum MP, Perez LE, Kristan WB (1986) A kinematic study of crawling behavior in the leech, Hirudo medicinalis. J Comp Physiol A 158:593-603

Thompson WJ, Stent GS (1976) Neuronal control of heartbeat in the medicinal leech. J Comp Physiol A Neuroethol Sens Neural Behav Physiol 111:261-279.

Tuthill JC, Wilson RI (2016) Mechanosensation and adaptive motor control in insects. Curr Biol 26:R1022-R1038.

von Holst E, Mittelstaedt H (1950) The principle of reafference. Naturwissenschaften 37:464-476.

Wolf H, Burrows M (1995) Proprioceptive sensory neurons of a locust leg receive rhythmic presynpatic inhibition during walking. J Neurosci 15: 5623-5636. 